\title{
Human olfaction: A view from the top
}

\author{
Stephen Pierzchajlo and Jonas K. Olofsson
}

Department of Psychology. Stockholm University, Stockholm, Sweden

Contact: Jonas.olofsson@psychology.su.se

\section{Acknowledgment}

This is a draft of a chapter that has been accepted for publication by Oxford University Press in the forthcoming book "Sensory Individuals" edited by Aleksandra Mroczko-Wąsowicz and Rick Grush due for publication in 2021.

\begin{abstract}
Although our understanding of human olfactory perception has increased dramatically, it remains less well understood how olfaction interacts with cognitive processes. In this overview, we review the claim that olfaction evolved to predominantly embody "novelty detection", a framework that leaves little room for cognitive representations and processes. We challenge this framework by pointing to several perceived limitations. Instead, we argue that human olfaction is best understood in terms of its reliance on top-down processes; visual or verbal contexts may generate predictions of odor qualities, and a fundamental role of olfaction is to evaluate such predictions. We hypothesize that olfaction is to a large extent dependent on "topdown" cognitive processes. We discuss how this framework differs from other contemporary approaches to human olfactory perception. We conclude that in contrast to other frameworks that characterize olfaction primarily in terms of stimulus-driven perception, human olfactory processing is best understood by invoking a "view from the top".
\end{abstract}

Keywords: olfaction; cross-modal; novelty; predictive coding; memory; perception 


\section{Introduction}

Whenever you enter someone's home for the first time, you are for a brief moment struck by how it smells; the unique molecular composition of lingering food odors, furniture and building materials, perfumes and body odors. Our ability to detect these abrupt changes in the olfactory environment constitutes perhaps its most distinctive feature in daily life. In the following, we will discuss how our mental representations of odor stimuli are generated at the interface between sensory stimulation and cognitive expectations. The research literature on olfaction has, arguably more than other senses, been concerned with the limitations of cognition and the dominance of bottom-up processes (e.g. Cain et al., 1998; Jönsson et al., 2005; Jönsson \& Olsson, 2003; Yeshurun \& Sobel, 2010). And while olfaction may indeed in some ways be cognitively limited relative to visual or auditory processing (Olofsson \& Gottfried, 2015), here we will argue in favor of a different viewpoint; that people are capable of using cross-modal cues to create predictive templates of odor input (i.e. patterns of neural activation generated by expectation), and that odor perception is to a significant extent created by matching sensory inputs to these templates (Zelano et al., 2011). Although olfactory research overall is arguably dominated by a stimulus-centered approach, we will be focusing especially on a "novelty detection" account (i.e. Köster et al., 2014), as it explicitly downplays the roles of cognitive representation of odor. We will describe the novelty detection and predictive coding frameworks, with a particular focus on how they are applied to olfaction. We will also evaluate key empirical and theoretical aspects of studying olfaction within these frameworks, and provide suggestions for future research.

\section{Novelty detection}

Distinct properties of stimuli and events are known to strongly influence attentional allocation. The visual system prioritizes stimuli that are large (Jonides \& Yantis, 1988), fast-moving (Treisman \& Gormican, 1988), or emotionally salient (Öhman et al., 2001). Likewise, fearful sounds (Kuraoka \& Nakamura, 2007), emotional human voices (Sander \& Scheich, 2001), and negative environmental noises (Zald \& Pardo, 2002) are all acoustically relevant features that characterize salient auditory stimuli. Unexpected stimuli represent another form of attentional capture, a process whereby stimuli that are unfamiliar/unexpected, either generally or in specific contexts, get prioritized for further processing via sensory-specific cortical pathways supporting attention (Ranganath \& Rainer, 2003). Given enough successive presentations or time spent processing a novel stimulus, increasingly habituating neural responses to the novel 
stimulus change their neural representational (Figure 1) from a novel to a familiar template (Kafkas \& Montaldi, 2018).

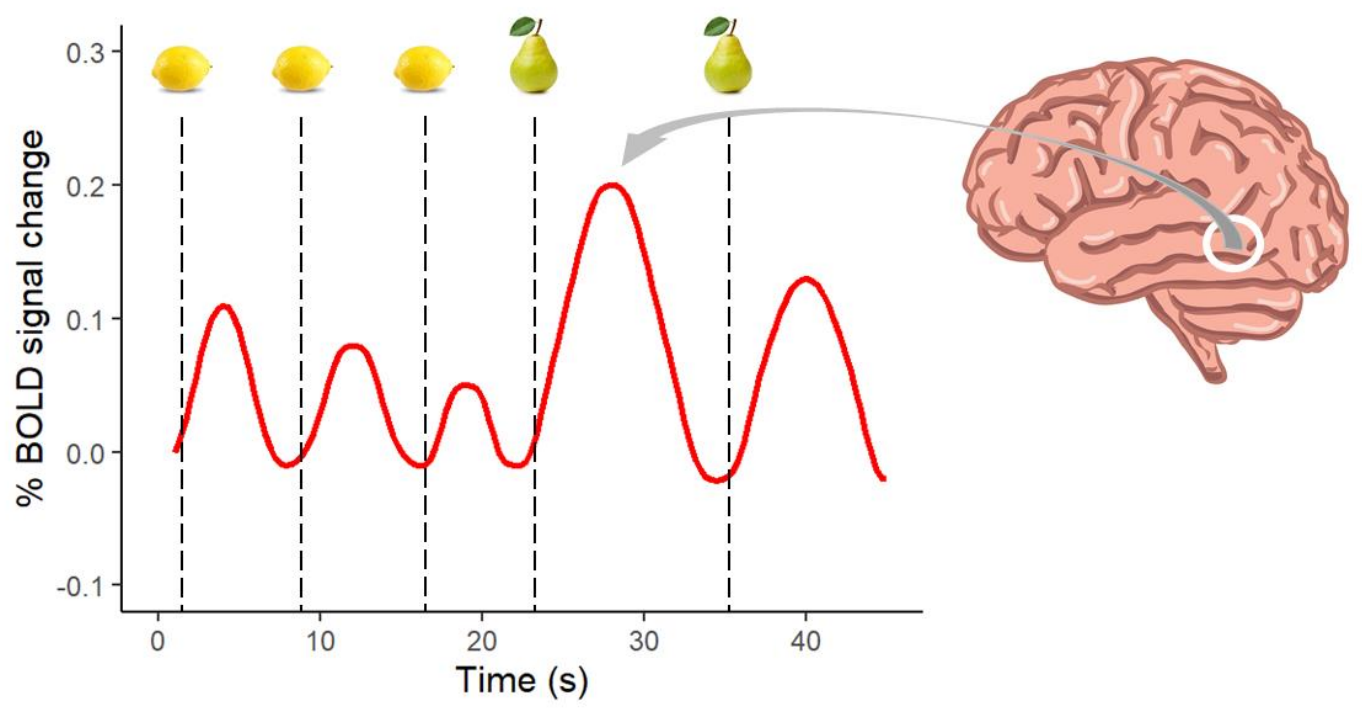

Figure 1: A schematic displaying the concept of novelty detection. In this hypothetical example, the red line represents the hemodynamic response function in the temporal cortex where visual objects are processed. The cortical response shows a reduced neural activation to a successively presented visual stimulus (lemon), followed by a relatively greater activation to a novel stimulus (pear).

Early neuroimaging research on novelty detection (Tulving et al., 1994) used positron emission tomography (PET) to record changes in neural activation from the visual cortex while participants viewed previously encoded (familiar) or novel images. By subtracting the PET activation of "new" images from "old" images, the authors' found several regions of the brain that were significantly more activated during this contrast, suggesting these regions were responsible for memory encoding when a visual stimulus was familiar. Upon further exploration of the data however, the authors were surprised to find that when they performed the inverse contrast (subtracting "old" images from "new" images), they also found areas of the brain that were significantly more activated for novel stimuli. Seemingly at odds with fundamental assumptions of memory research at the time, the hippocampus (an area vitally important for memory) was significantly less active when participants were presented with familiar images compared to novel ones. As the authors put it, “... what we had observed was that remembering past experiences (reduced) the activity in the hippocampus, the 'seat of 
memory' (Tulving et al., 1994)". Around the same time, several single-cell recording primate studies were finding neurons that were more activated by "new" pictures compared to pictures the monkey had seen many times (Fahy et al., 1993; Li et al., 1993). By combining insights from the primate literature with their puzzling fMRI data regarding human hippocampal activity, they reasoned that what they were viewing was not the "deactivation" of the hippocampus to familiar stimuli, but rather, its "activation" in response to novel stimuli (Tulving et al., 1994). This suggested that perhaps the hippocampus served memory functioning not by detecting what was familiar, but rather, by detecting what was unfamiliar (Tulving, 2010).

The aforementioned PET study, and other studies with similar results (Fahy et al., 1993; Li et al., 1993), led Tulving and colleagues to propose the "novelty/encoding hypothesis" (Markowitsch et al., 1996; Tulving \& Kroll, 1995) to explain the mechanisms related to these supposed novelty-related responses. Their hypothesis postulated that novel visual stimuli are first detected by the hippocampus/limbic system, triggering complex higher-level encoding operations. Through this process, visual information that is identified as novel is further analysed prior to memory storage while familiar (i.e. non-novel) visual stimuli are stored without additional processing. Several competing theories have since tried to capture potential mechanisms used by the brain to first determine whether a stimulus is novel, and then to further process stimuli determined to have such qualities. Early popular novelty detection theories, like the comparator/match-mismatch model (Kumaran \& Maguire, 2007) described novelty detection as a comparison between incoming information and prior representations already stored in the brain. For example, Kumaran and Maguire (2007) hypothesized that "the hippocampus may generate predictions about how future events will unfold, and critically detect when these expectancies are violated, even when task demands do not require it". Shortly after, a different notion of novelty detection was formulated by Tulving (2010). He argued that the process of novelty detection could theoretically occur without any of the match-mismatch mechanisms proposed by authors like Kumaran and Maguire (2007). To elaborate this point, Tulving (2010) first hypothesized a state of the brain where neural networks are engaged in the processing of a repeated sensory stimulus. Repeated engagement of these specific networks automatically attenuates their activation. The sudden presence of a new stimulus would engage new neural networks whose activation had not yet been repeatedly engaged, and thus, these networks would naturally have higher activation levels. It is therefore not the detection of a stimuli's novelty value that amplifies neural signaling and memory encoding, but rather, it is 
the automatic engagement of new patterns of neural activation that cause a rise in neural activity in the presence of novel stimuli (see Tulving \& Rosenbaum (2006) for a detailed example). Thus, while novelty detection does exist as a human behavioural operation, there is no requirement for specific novelty encoding neural operations in the brain, as these findings can be explained simply by assuming that familiar stimuli are processed in a non-distinctive way, leading to observable activation increases in the presence of novel stimuli.

The processing of novel stimuli is often studied under a paradigm of repetition suppression, whereby stimuli that are repeated many times result in the decreasing amplitude of their corresponding neural responses (Ringo, 1996). This decreased responding corresponds to the gradual decrease of the stimuli's novelty value. Another novelty-related concept, adaptation, deals with the reduction in the cortical response of neurons to stimuli that have been previously experienced. Repetition suppression experiments are thought to be based on adaptation, and its common characteristics include short response times and a relative automaticity in the response. Similar cellular mechanisms might be involved, although the specific location in the brain depends on the exact feature being adapted to. For instance, ferrets presented with rapidly appearing contrast gratings were found to have attenuated action potentials in the primary visual cortex, a processes driven primarily by changes in the calcium-dependent potassium current of the neurons (Sanchez-Vives et al., 2000). Conversely, Sohal and Hasselmo (2000) used a computational model to reason that, based on single-cell spikes recorded from the inferior temporal (IT) cortex in monkeys viewing familiar stimuli, the IT habituates to familiar visual stimuli via reduced calcium-dependent action potentials.

More recent novelty-related work has highlighted brain regions like the medial temporal lobes (MTL; including the hippocampus, perirhinal cortex, entorhinal cortex, parahippocampal cortices, and amygdala) as being crucial for novelty detection. For instance, processes involving the cooperation of regions within this network have been shown to generalise to many different classes of stimuli including novel words, objects, scenes, (e.g. MTL structures; Kafkas et al., 2017) and novel faces (e.g. amygdala; Staresina et al., 2012). Additionally, decades of EEG research have established components like the P300 that are elicited by both context-dependent and context-independent novel (i.e. infrequent) stimuli (Sutton et al., 1965). Similar effects are found in human and non-human mammals (Paller, 1994). Novelty responses do not necessarily confer a behavioral advantage, as is exemplified by a study using picture stimuli (Olofsson \& Polich, 2007). Here, participants were to respond via button-press to picture target stimuli that 
were inserted into a sequence of identical, non-figurative standard stimuli. Each unique target was presented four times before shifting to a new target, but the number of interleaved standard stimuli varied and thus the target onset could never be exactly predicted. Responses to novel targets were associated with a smaller and slower P3, as well as delayed behavioral response times, compared to when the same targets were repeated subsequent times; this result suggests an improved cortical and behavioral classification of familiar picture targets (Olofsson \& Polich, 2007).

Importantly, the novelty P3 is regarded as modality-unspecific and generalises both to vision and touch (Knight, 1996). When different sensory modalities are studied under similar conditions, odor stimuli elicit a P3 component that is highly similar to the P3 elicited by auditory and visual stimuli, and the amplitudes of ERPs elicited in these three senses are similarly diminished when top-down attention is directed away from the stimuli (Andersson et al., 2018). An important conclusion from the novelty detection literature is that the concept of novelty does not just refer to unfamiliar stimuli; even familiar stimuli that are surprising in the given context can be considered novel, and processed accordingly. Another conclusion is that novelty response increases do not necessarily confer a behavioral advantage, but that modalitygeneral mechanisms, such as attention and stimulus expectancy effects can be expected to influence olfactory processing.

\subsection{Olfactory novelty detection}

How does novelty detection apply to the olfactory system? The most developed account of olfactory novelty detection is, to our knowledge, that of Köster and colleagues (2014). The foundation of this account is the prominent sensory adaptation and habituation observed in the olfactory system. Odor exposure rapidly leads to a decreased sensitivity for the stimulated odor, and to some extent other odors. While olfactory adaptation varies across odors, researchers generally agree that factors like odor concentration and exposure time greatly contribute to adaptation effects (Dalton, 2000). Depending on the magnitude and duration of the olfactory stimulation, recovering odor sensitivity might take several minutes. Early research also showed that stimulating one nostril with an odor dampens sensitivity in the other, even though their corresponding olfactory neuroepithelia are anatomically separated and project to different cerebral hemispheres (Elsberg, 1935). This led researchers to realize that central mechanisms were partly responsible for the decreased sensitivity, and called this central effect "habituation" which was distinguished from sensory-level "adaptation" (Köster \& de Wijk, 1991). The 
novelty detection account that we will focus on here, may be viewed as a generalization of findings from olfactory adaptation and habituation research to account also for other odor memory research findings. According to the novelty detection account, we typically experience odors implicitly and without much conscious attention. The sense of smell functions mainly as a warning system that alerts us of the presence of potential airborne pathogens, or stops us from ingesting dangerous food. Familiar or expected odors are thus ignored, and only novel or unexpected odors are noticed. It is well known that healthy elderly individuals often do not notice that their sense of smell is diminished (Nordin et al., 1995), whereas blindness or deafness does not normally go unnoticed. According to Köster et al. (2014), another indication that olfaction does not seem to prioritize known odors is the fact that olfaction is a sense with “complete adaptation”. Several studies (e.g. Köster \& de Wijk, 1991) have indeed found that sensitivity to a presented odor is lost after only a few minutes of exposure, and it may take up to several minutes to return to baseline sensitivity. Conversely, full visual adaptation does not occur because, the authors argue, visual object representation is always important for spatial navigation and object identification (Köster et al., 2014). Köster et al. (2014) thus formulated the "misfit theory of spontaneous conscious odor perception" (henceforth called "misfit") to outline what they perceive to be olfaction's major (or potentially only) contribution to human perception.

To investigate the misfit framework outlined above, we first need to define "odor identification"; traditionally, it involves explicitly knowing the odor source. While such knowledge can be achieved in principle with or without contextual cues (Schab, 1991), most researchers nowadays distinguish between "odor naming", when the odor source is generated without any supportive cues, and "odor identification", associating an odor with a non-olfactory (e.g. written, spoken, or depicted) response alternative that describes its matching source. Correct odor identification performance thus involves selecting matching stimulus pairs and refuting mismatching pairs. Odor identification is considered a type of declarative, explicit, knowledge-based odor memory, and it is incorporated in standardized olfactory assessments (Oleszkiewicz et al., 2019). The distinction between identification and naming is important to retain because odor naming is much more difficult than identification, and confusing the two tasks may cause confusion about the nature of human olfaction (Olofsson \& Pierzchajlo, 2021).

According to the misfit framework, odor identification and naming should not be a crucial function of human olfaction. Instead, the authors argue that odors are typically encountered 
non-consciously and are thus processed as implicit associations about situations, rather than involving knowledge of odors themselves. Odors that fit these associations are thus not consciously evaluated because the aforementioned implicit associations about the presence of the odor are already met. "Misfits" only occur when there is a mismatch between the implicit association between the odor (or odors) and the present situation. However, only contextually deviant odors demand cognitive processing, whereas expected odors remain implicit and unnoticed (Köster et al., 2014). Importantly, their argument implies that perception of odors does not require olfactory objects. This conceptualization also seemingly denies the existence of any perceptual influence from higher-order states, such as predictions based on explicit cues, on our perception of olfactory stimuli. Olfactory perception is hence viewed as being driven by bottom-up change detection processes. Köster and colleagues question the need for an explicit odor identification/representation system and argue that research on explicit odor perception and memory abilities is inherently flawed (Köster et al., 2014).

The strongest evidence in support of the misfit framework (Köster et al., 2014) comes from a specific food memory paradigm. In this paradigm, participants incidentally encode odor memories during a breakfast without knowing their memory of the odors will later be tested. Participants are later presented with odors or tastes of food items that either were (target) or were not (distractor) present at the breakfast and must try to correctly remember as many targets as possible. The authors claim that results from such experiments demonstrate that even though we have weak abilities to recognise odors that were previously present, we are simultaneously very proficient at determining when an odor was not encountered before. The authors argue, based on the results, that representations of the identities of odors are not important for olfactory recognition. As Köster et al. (2014) put it, "what is not there ... cannot be interfered with or forgotten, but novelty is always functional". Because the authors argue that memory traces of previously encountered olfactory stimuli are not present, we take this view as the authors' suggesting that odor identities are not represented in the mind.

We argue that although the misfit account of olfaction captures some important characteristics of odor perception, the account can be questioned on empirical and theoretical grounds. Our concerns are outlined in the following sections. The framework was evaluated by MorinAudebrand and colleagues (2012). In their meta-analysis, they claim to demonstrate the robustness of the key results of the food memory paradigm. Specifically, Morin-Audebrand et al. (2012) synthesized results from 6 research articles (Koster, 2004; Laureati et al., 2008; Mojet 
\& Köster, 2005; Moller et al., 2007; Morin-Audebrand et al., 2006; Sulmont-Rossé et al., 2008) using the novelty detection paradigm as it is considered a key source of evidence for the misfit framework (Köster et al., 2014). In all 6 meta-analysed studies, participants were recruited under the guise of participating in a study about individual predispositions towards food. At some point during the experiment, breakfast or lunch was served to allow for incidental learning of the food stimuli participants would later be tested on. A consistent result in all studies is that participants have difficulty determining which odors and tastes of food were present during the meal, and are instead more accurate at determining the odors and tastes that were not present (Figure 2). Morin-Audebrand et al. (2012) argue this demonstrates that olfactory memory is not concerned with storing representations of odor objects, and instead evolved to detect odors that are novel.

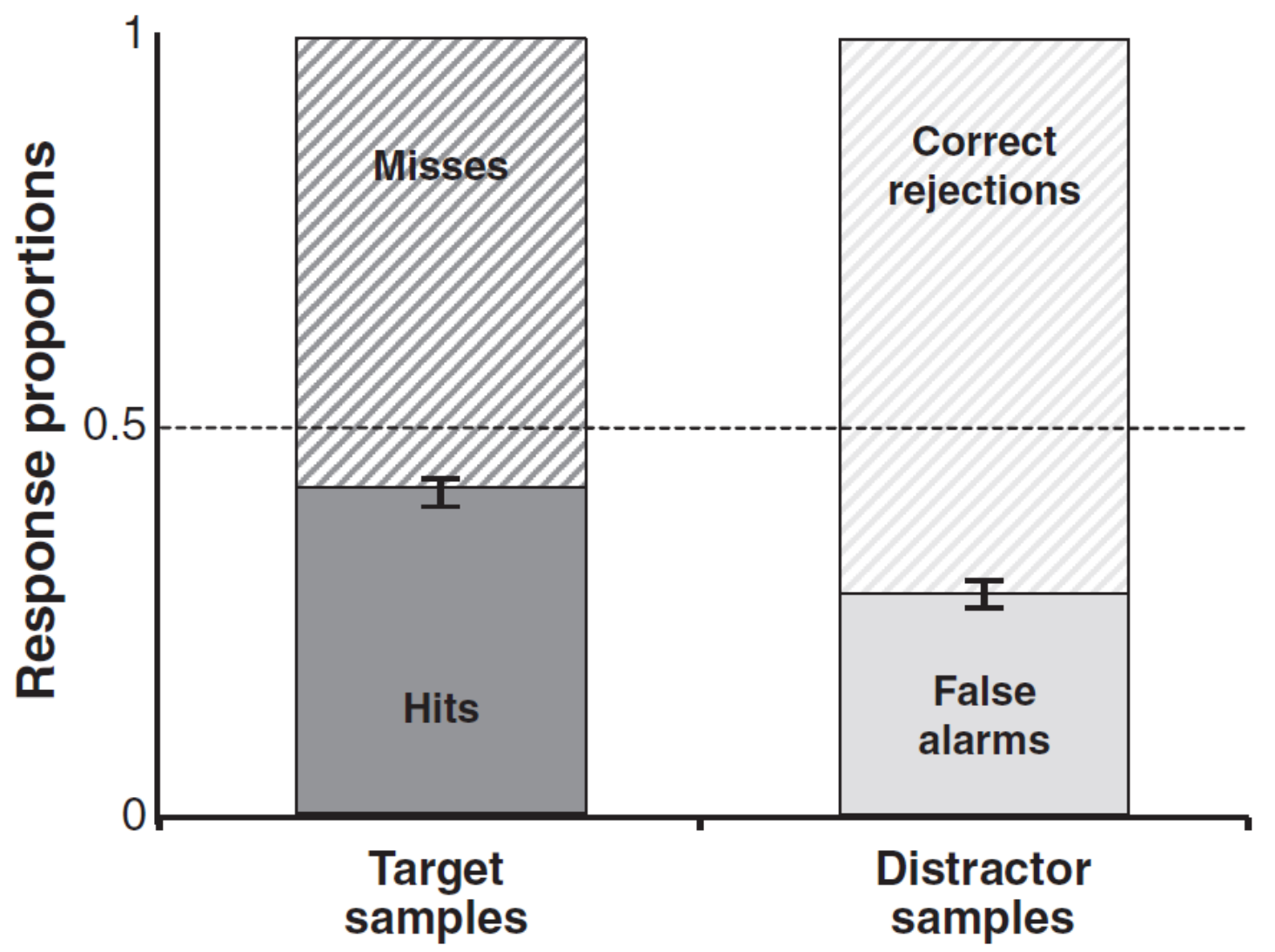

Figure 2: Combined data from the 6 studies cited in the meta-analysis by Morin-Audebrand et al. (2012). On average, participants are better at determining when an odor was not previously present (correct rejections) compared with their ability to determine when an odor was previously present (hits). Adapted from Morin-Audebrand et al (2012) with permission. 


\subsection{A critique of the misfit account}

According to the misfit account of olfactory novelty detection, olfaction does not store object representations and instead, olfaction is almost exclusively concerned with processing novel stimuli (Morin-Audebrand et al., 2012). We argue that the evidence provided is insufficient to downplay the importance of cognitive olfactory representations. In general, in order for a theoretical notion to be considered true, converging evidence needs to be presented from a range of experimental designs (Yarkoni, 2020). Instead, the misfit framework is formulated as a general theory of human olfaction, but the fundamental claim that olfactory cognition prioritizes novel over familiar stimuli relies on evidence from a specific experimental design. However, the key finding of better performance for "correct rejections" compared to "hits" (Figure 2) is not found in other types of odor memory experiments. In fact, traditional recognition memory experiments with odor stimuli show that participants often have an unusually high "hit rate" (recognizing that an odor was "old", i.e. presented during a prior encoding phase), but that novel odors are often mischaracterized as "old"; in fact, some experiments show that the recognition of odors is more prone to such outcomes than the recognition of faces, and that odor recognition memory is further facilitated when the odor is familiar (e.g. Cornell Kärnekull et al., 2015). These results suggest that the misfit account of olfaction might not generalize to the odor memory domain.

The authors of the misfit account seemingly do not acknowledge a distinction between sensorylevel and contextual novelty, a distinction we regard as important. Novelty detection at a sensory-processing level is a key bottom-up perceptual mechanism which we believe is unquestionably important for the olfactory system. Here we agree with the authors of the misfit account. However, contextual novelty (i.e. where an odor does not fit our expectations about the environment) is described by the authors as a bottom-up process (Köster et al., 2014), when we believe it should in fact be conceptualised as a top-down mechanism - an expectation that is violated by an incoming odor stimulus. Thus, while the novelty detection framework denies olfactory objects as an existent entity, in the following sections we will instead argue for an important role of cognitive "top down" processes, and how they can interact with olfactory stimuli to influence how we perceive them. We will highlight experiments that seek to establish or assess expectations (or other cognitive representations) in the olfactory system. These experiments consistently generate results that we believe are at odds with the misfit account, but consistent with an alternative, predictive coding framework. 


\section{Predictive coding}

Already in the early days of psychological science, Hermann von Helmholtz (1860) reasoned that perception is a probabilistic process where sensory systems attempt to infer the causes of bodily effects. His work influenced later scholars (i.e. Gregory, 1980; MacKay, 1956; Neisser, 1967) whose combined contributions can be summarised using an analysis-by-synthesis paradigm (Yuille \& Kersten, 2006); the brain does not build models of the world by piecing together bottom-up information, but instead tries to predict current sensory inputs from a list of possible causes (i.e. models that can be compared). Predictive coding describes a process whereby higher-level brain areas in a sensory hierarchy attempt to predict inputs occurring at lower-levels on the basis of their imperfect model of the world (Clark, 2013). These predictions are compared to the actual activation in lower level sensory circuits. This comparison typically results in some level of discrepancy between the prediction at the higher level and actual input generated at the lower level. The prediction error (predicted cause - actual cause $=$ error) is summed and transmitted back up the hierarchy to the level where the prediction originated (Rao \& Ballard, 1999) activation, no alarm is raised and no additional processing occurs. Should a discrepancy occur, however, the higher cortical level uses this error as feedback and attempts a reformulation of its current model by updating its prior probability of the sensory data occurring at the level where the prediction error was made. This process is repeated iteratively and continuously until the discrepancy between prediction and sensory input have been sufficiently minimized. Such computational error correction mechanisms are, according to this framework, central to mammalian brain function.

How then do prior expectations about stimuli shape perception, and how might those mechanisms operate? Expectations about the world are known to bias perception, as evidenced in visual illusions; for instance, the light-from-above prior makes circles appear concave or convex depending on where a shadow is cast (Oliva \& Torralba, 2007). Because certain features appear more frequently in natural environments, expectation mechanisms allow organisms to form prior probability models about events/stimuli that can aid their survival (de Lange et al., 2018). Prior probability models are therefore likely encoded in the neural tuning properties of the sensory cortices themselves (Cloherty et al., 2016). For example, cardinal (horizontal and vertical) orientations are more prevalent than other orientations in natural images and, unsurprisingly, visual neurons that are tuned for recognizing cardinal orientations are most represented in the early visual cortex (Albright, 1989). Additionally, prior experience within a specific context can give an ambiguous stimulus a more concrete identity. For instance, an 
otherwise amorphous blob can appear to be the Statue of Liberty when appropriately placed in an image of Manhattan and its surrounding rivers (Lavenex \& Amaral, 2000).

\subsection{Cross-sensory predictions in olfaction}

There is strong evidence that predictive coding mechanisms are present also in the olfactory system, and such predictions are utilized in olfactory perception and cognition processes. Some olfactory capabilities (i.e. object detection and recognition) are much slower than their visual analogues; for example odor identification is estimated to 600-800 $\mathrm{ms}$ for olfactory identification (Laing \& MacLeod, 1992) versus in vision, object identification takes about 250 ms (Robinson, 1968). However, it is a well-known fact that supporting visual information is greatly beneficial for enhancing performance in olfactory tasks. That human olfaction might functionally rely on other sensory systems (i.e. vision) to make up for its limitations in identification is not a new idea. According to the "primitive olfactory brain" hypothesis, building on the pioneering work of the comparative neurologist Charles Judson Herrick (Herrick, 1933), mammalian olfaction strongly relies on cues from non-olfactory sensory modalities to competently perform olfactory tasks, such as identifying the source or location of an olfactory stimulus. Evolutionary development introduced a gradual brain growth in human ancestors, but olfactory cortex declined relative to the total brain size (Moulton, 1967). Thus, olfactory representations and the physical size of the olfactory cortex in primates became dramatically overshadowed by the neocortex, a consequence of evolution favoring higher cognitive functions and multisensory associations over sensory-specific analysis (Ribeiro et al., 2014). Herrick (1933) hypothesized that the aforementioned interconnectivity of mammalian olfactory and non-olfactory sensory regions might point to a perceptual strategy unique to olfaction, namely, that mammals to a large extent use information from other sensory systems to enhance olfactory identification.

The piriform cortex (PC) is involved in the encoding of odors; often, this process involves configural representations of odor objects whereby individual molecular odor components are not perceptually separable, but instead they are represented by a total sensory object (ThomasDanguin et al., 2014). Odor objects are conceived of as a form of perceptual memory representation, where neural responses to the many constituent odor molecules and features are associated and integrated through a learning process that eventually results in a holistic percept (Wilson \& Stevenson, 2003; Wilson \& Sullivan, 2011). Molecular and experiential features both contribute to varying degrees of configurality in the perception of molecular odor mixtures 
(Thomas-Danguin et al, 2014). Conversely, visual stimuli can be processed in terms of their individual perceptual components because the visual system encodes stimuli in a hierarchy of visual associative areas (Singer \& Gray, 1995; Tanaka, 1996). Relative to a visual object, olfaction retains only few robust perceptual features by which stimuli can be individualized (e.g. Valence: Khan et al., 2007; Edibility: Zarzo, 2008). Such differences might help explain why odors are often extremely difficult to identify in the absence of any other sensory information (Cain, 1979; Engen \& Ross, 1973; Laing \& Francis, 1989; Livermore \& Laing, 1996).

How might novelty detection accounts be empirically differentiated from predictive coding accounts? We believe that response-time studies with predictive cues provide useful evidence. In a response-time study supportive of the novelty detection account, Möller and colleagues (2007) reported that when two odors are presented in succession and participants are asked to decide whether they were the same or different, the different odor pairs were detected more rapidly than when the odors were the same. The results were interpreted as supporting the preferential processing of novel odors (Moller et al., 2007). In our view, these results are likely influenced by the sensory adaptation that occurs when the same odor is repeatedly presented. The first odor, de-sensitizing the olfactory receptors to the following stimulus will cause a delayed response when the odor is presented a second time. Sensory adaptation explains parsimoniously why different odor pairs are more rapidly detected. Thus, this empirical result does not say anything about the existence or roles of mental representations of odors or their use in perceptual predictions, and it only supports the well-known phenomenon that olfactory sensitivity diminishes by repeated stimulation.

If novel odors were indeed prioritized behaviorally, as suggested in the misfit account, odors that are unexpected given the experimental context would be rapidly processed when sensory adaptation was eliminated as a potential confound. However, the opposite result is typically observed. Cross-modal cue-target experiments have repeatedly shown that when participants are primed with a word (e.g. "lemon"), they are faster in responding "yes" to a subsequent matching odor target odor (lemon) relative to responding "no" to a non-matching (and hence contextually novel) target odor (e.g. rose; Olofsson et al., 2012, 2013). In these experiments, participants do not have to retrieve the identity of the odor upon perceiving it (which varies across trials), just indicate whether the percept matches or not with the expected odor percept, which was indicated by the identity cue. Inconsistent with the novelty account of olfaction, 
there is an sizable delay (>100 ms) for unexpected (contextually novel) odors. Importantly, the novelty-detection framework would predict the opposite results; to the extent that people can use cross modal cues to predict odor input, an incongruent (novel) odor would be processed faster even when primed by a non-olfactory stimulus such as a written or spoken word. Here, the experimental setup avoids the risk of confounding sensory adaptation because the cue is presented in another sensory modality than the target. Furthermore, the cross-sensory cue-target paradigm has an advantage in that the same cues and targets are presented in every condition with only their congruency varying, minimizing risk of stimulus bias. The advantageous behavioral processing for expected odors is evidence that participants understand the word cue (e.g. "lemon"), translate its meaning into a perceptual template of lemon odor even before the odor is perceived, and use this prediction to facilitate the processing of the odor. This result would not be observed if the olfactory system had a general tendency to prioritize novel odors.

Cross-sensory cue-target experiments provide a method of comparing olfactory processing efficiency in terms of response-times, and they can be modified in a flexible way to study a variety of features. For example, researchers may alternate the sensory modalities that constitute cues and targets, vary the stimulus set size, the cue-target delay, the withinexperimental probability of certain items, and/or the features that are prompted by the cues. Research within this experimental paradigm has shown that odor source objects (e.g. "lemon") are particularly effective in facilitating rapid and accurate responses, whereas cuing based on odor valence or edibility are less effective, suggesting that the odor object may constitute a fundamental unit of perceptual processing (Olofsson, 2014). When odors are instead used as cues for a subsequent verbal target (e.g. a lemon odor cue primes the participant to a target word that can be either "lemon" or another word in the set of stimuli), the word target appears to reactivate olfactory-associated brain regions when the target is unexpected, seemingly to provide prediction error feedback to the associative olfactory cortical regions where the prediction was made (Olofsson et al., 2014). When odor cues are non-matching with the word target there is a behavioural delay, suggesting, again, a need for more extensive processing of non-matching combinations (Olofsson et al., 2014, 2018). Overall, our experiences from cross-modal studies of cue-target matching suggest that congruent trials are always faster than non-matching trials. It should be noted that under some circumstances, this rapid processing might come at the expense of decreased accuracy (Olofsson et al., 2014) but the speed-accuracy trade-off is not always observed (Olofsson et al., 2018). All our studies show a speed advantage for congruent cross-modal odor stimuli. In a recent study, Pierzchajlo et al. (2019) let participants determine 
whether or not a verbal cue matched a subsequent visual or olfactory target stimulus. We found that the greatest disparity in response time differences between congruent and incongruent cuetarget combinations occurred when the word cue was object-based (e.g. "lemon") and the target was olfactory, rather than visual (Figure 3). Although more research is needed, this result suggests that the olfactory system may be even more dependent on predictive codes (from, in this case, a word that cues perceptual objects) than the visual system, at least in object identification tasks. These results are in line with the notion proposed first by Herrick (1933), that the human olfactory system compensates for the lack of an extensive olfactory cortex by effectively using information from other senses in order to identify an odor. This influence, we argue, comes in the form of cross-modal predictions that are imposed on the olfactory cortices.

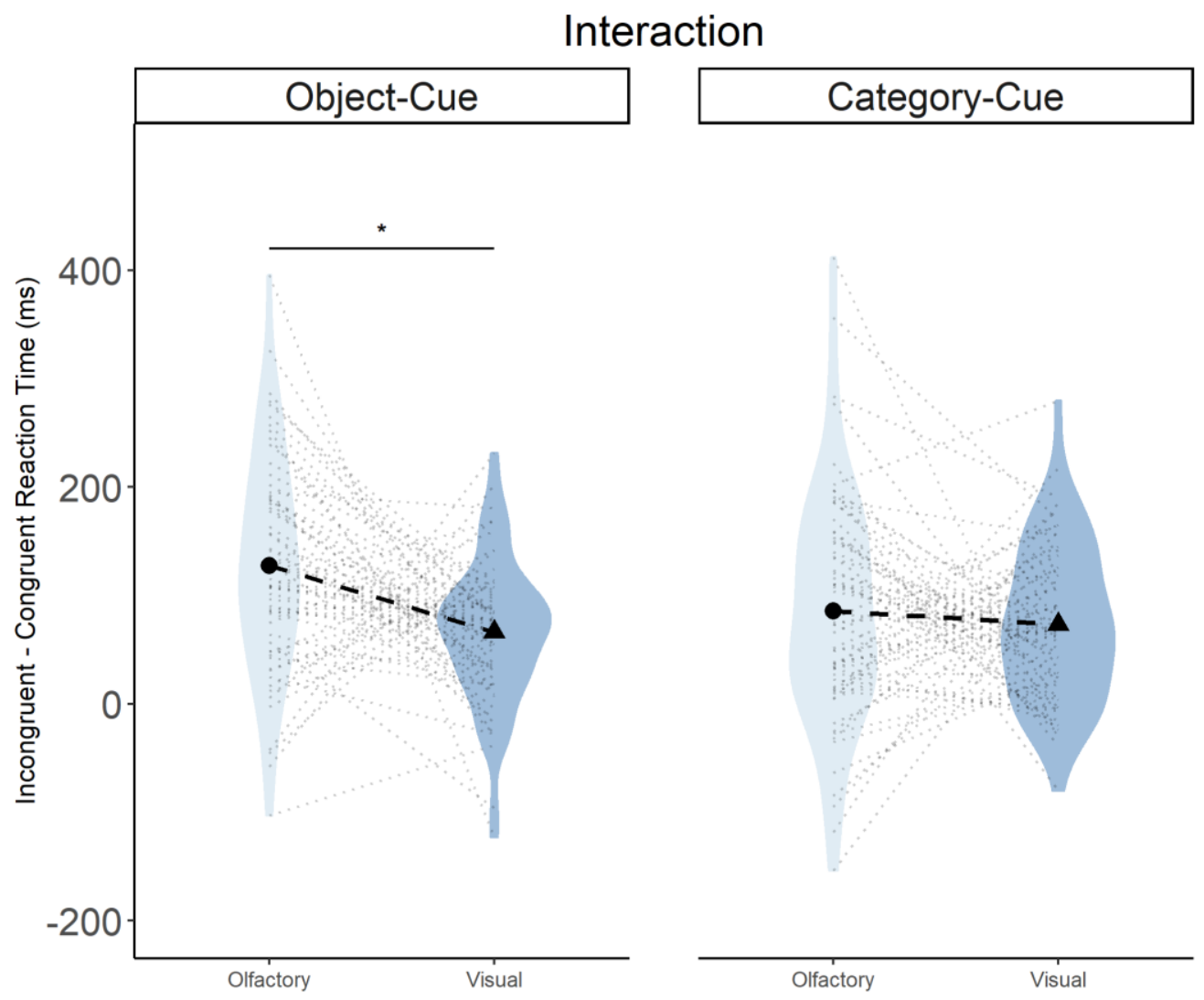

Figure 3: Difference between incongruent and congruent reaction times (ms) for the objectcue and category-cue conditions as a function of target modality. All conditions indicated a "novelty delay" for cue-incongruent odors. In the object-cue condition we found a significant interaction between modality and congruency such that the greatest disparity between incongruent and congruent reaction times was observed during olfactory target trials. This indicates odor object predictions are especially important for olfactory perception in this task. No such statistically significant interaction was observed during category-cue trials. 


\subsection{Olfaction as "a view from the top"}

Recent theoretical work suggests that the combination of PC representational coarseness, coupled with known top-down feedback connections from other cortices to the PC, indicates a route for odor-predictive visual information to enhance olfactory identification (Olofsson \& Gottfried, 2015). The PC is considered to be critical for establishing configural odor percepts (Gottfried et al., 2006; Howard et al., 2009). In the absence of any other information, olfactory stimuli presumably activate a set of potential odor identities because the relatively coarse, configural nature of olfactory responses does not allow precise and multifaceted odor representations. Conversely, because visual stimuli are processed in an elaborate hierarchy of feature-selection regions of the visual cortex (Distler et al., 1993; Saygin et al., 2012; Van Essen et al., 1992), the identity of a visual stimulus is rarely ambiguous. However, because the ventral stream of the visual cortex contains feedback projections to various olfactory regions, Olofsson and Gottfried (2015) hypothesised that rich visual information could be used to communicate predictions to the PC via these feed-backward pathways (Figure 4). Thus, an anticipatory template in the PC for a potential odor could be generated via congruent visual or auditory information that allows the PC to increase the probability of successful odor-object identification by simply generating a pattern of activation that represents an analogous olfactory representation (see Zelano et al., 2011). Importantly, these patterns of activation can hold for a few seconds during an anticipatory period prior to the onset of an olfactory stimulus, and decay quite rapidly, especially when the odor source does not match the neural template (Zelano et al., 2011). Recent fMRI evidence suggests the posterior part of the PC functions as a "multisensory binding site" where auditory and visual cues are integrated with concurrent odors, increasing its activity level as a function of the number of stimulated non-olfactory input channels (Porada et al., 2019). Recent evidence also suggests that multimodal integration is mediated by the phase-locking of low-frequency waves across brain regions. For instance, Zhou et al. (2019) found that when verbal cues were used to predict odor target stimuli, neural oscillations occurring in both the PC and auditory cortex became phase-locked (i.e. carried matching oscillatory synchrony) in the prediction phase of trials, but only on trials where participants were able to correctly assess whether the cue and target matched. Interestingly, this result suggests not only a cortical mechanism underlying predictions in the olfactory system, but also suggests a role for direct input from other perceptual regions. Several aspects of this process remain to be elucidated, including the possibly complementary roles of sensory and 
associative cortical networks. Future research may provide a more detailed account of how predictive processes are generated and imposed on the olfactory cortex.

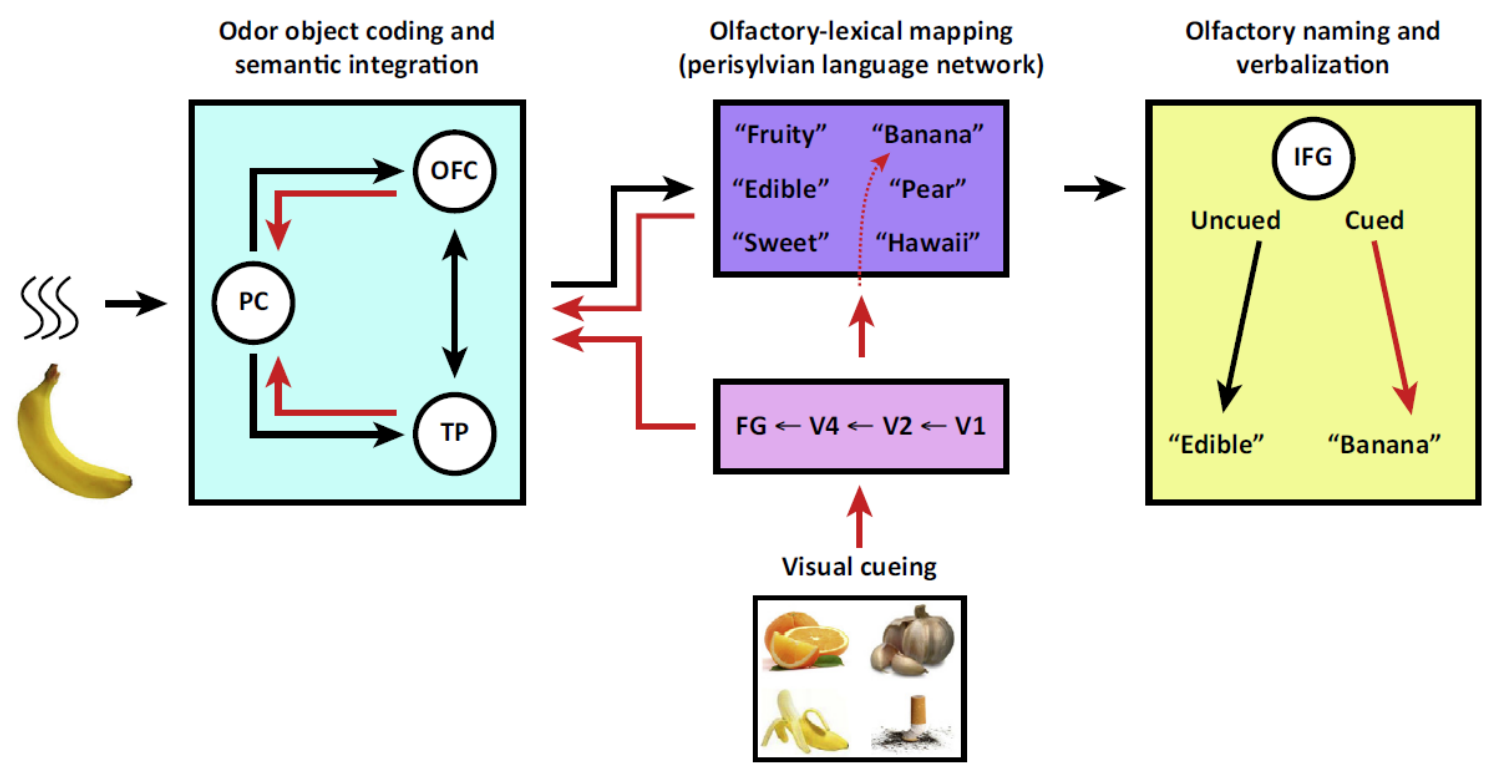

Figure 4: A model outlining how predictive cues from competent stimulus identification senses (i.e. vision) can allow enhancements of the same processes in less competent stimulus identification senses (i.e. olfaction). Taken from Olofsson and Gottfried (2015) with permission.

\subsection{Novelty Detection or Predictive Coding?}

How do we reconcile our account with the empirical finding that people are better at recognizing "new" compared to "old" (previously presented) food items in a flavor recognition task? We note that the food recognition task design does not involve any specific predictions or mental simulations of the stimuli. Instead, studies using this paradigm involve a stimulus input that is matched with memory representations. Thus, a key distinction between novelty and predictive coding accounts is that the "misfit" framework only recognizes odors bottom-up from a bottom-up change detection. While we agree olfaction certainly works this way, we argue that olfaction also possesses a top-down cognitive capacity that is best understood through systems predicting incoming odors by using representational templates to aid successful identification. Under circumstances tested within the "misfit" framework, in the absence of robust contextual predictions, it is possible that novel odors/flavors attract more processing resources than familiar odors/flavors. But such features of novel stimuli might also 
be observed in visual and auditory novelty research, as we have reviewed, and it does not necessarily point to novelty processing being a unique feature of olfaction. We are, in fact, not aware of any findings in memory research that would decisively show that novel odors result in enhanced mnemonic processing beyond what is observed in other sensory modalities. Additionally, Pierzchajlo et al. (2021) show that, as shown in Figure 3, that people are faster at responding to when an odor matches a verbal cue than when it does not. These results do not fit with the general claims of novelty as a dominant feature in olfaction; instead, they might be viewed as supporting the notion that olfactory processing is highly dependent on cognitive memory representations.

We conclude that the misfit account for olfactory novelty detection is based on the well-known perceptual tendency of olfaction to rapidly decrease in perceptual sensitivity during repeated or prolonged stimulation. However, this phenomenon is, in our view, over-generalized to the cognitive domain, where it does not fit with several lines of available evidence. Cognitive olfactory processing is instead highly dependent on prior experiences and contextual predictions, making cross-modal cues and memory traces effective facilitators of performance. However, we think that one cognitive aspect of the novelty detection framework is accurate; the general tendency for novel, unexpected, or contextually incongruent odors to attract attention and other processing resources. We have found that incongruent odors demand more elaborate and effortful processing, sometimes at the expense of processing visual stimuli in a multisensory environment (Hörberg et al., 2020). But importantly, olfactory novelty does not typically confer a behavioral advantage, and instead it requires effortful and slow processing.

\section{Conceptual distinctions in olfactory novelty and predictive coding research}

When olfactory researchers write about stimulus novelty, there is sometimes not a clear operational definition of terms that specify what novelty entails, and authors typically use novelty as an umbrella term. To differentiate between frameworks emphasizing novelty and prediction error, it is important to distinguish the key concepts. From our understanding, it seems that the term novelty is most often used to refer to a distinction between familiar and unfamiliar stimuli. Multiple review articles (Kafkas \& Montaldi, 2018; Ranganath \& Rainer, 2003) refer to the contrast of "familiar versus novel" which indicates that novelty has to do with an assessment of a stimuli's familiarity. For instance, both repetition suppression and adaptation studies (that involve "novelty" assessments) measure responses either to a stimulus the participant is already familiar with, or to a stimulus that the participant is becoming familiar 
with. However, when talking about novelty in the context of other paradigms, like event-related potentials experiments, the crucial distinction regarding what makes a stimulus novel is instead whether it matches the context of the experiment. In such a case, it may be more appropriate to say the stimuli are deviant, rather than novel. It seems that researchers studying olfactoryrelated novelty detection also often tie novelty to contextual parameters rather than familiarity. In fact, the mock-breakfast paradigm outline by Morin-Audebrand (2012) and colleagues seems to largely depend on operationalizing novelty in terms of context. Breakfast items do not typically represent unfamiliar food items, as most people are very familiar with typical breakfast foods. Instead, the novel breakfast items represent foods the participant did not eat at the breakfast. In this context, it makes sense to think about this aspect of olfaction as being related to context-dependent novelty, rather than familiarity. In fact, authors of several papers even try to use targets and foils that are as similar as possible in order to remove the familiarity of stimuli as a confound (i.e. Mojet \& Köster, 2005). Thus, these paradigms appear to capture "novelty" only in the sense that the novel stimuli are novel to the participants in that specific context, and the familiar stimuli are familiar in that specific context. These paradigms do not capture expectation violations, however, as there is no reason to believe participants only expect to encounter food they previously ate. Complementary approaches are thus needed to study different aspects of novelty and deviancy.

Predictive coding effects do not seem to be driven by prior familiarity of the stimulus, as they are similarly observed for familiar and unfamiliar stimuli, nor do they rely on contextdependent assessments, and they appear to be generated quite automatically. In many predictive coding paradigms, regardless of the behavioral task, expectation violation is typically what drives the effects. In such cases, there is an expectation based on prior sensory information, which is violated (i.e. Egner et al., 2010). One additionally important feature of predictive coding paradigms is that unexpected stimuli achieve their distinction of unexpectedness through their rare occurrences. For instance, Both Kok et al. (2012) and Egner et al. (2010) only presented the unexpected (expectation violating) stimulus on $25 \%$ of trials (versus $75 \%$ for expected stimuli). Finally, the misfit account of olfaction is not systematically supported by research on explicit expectation violations or relative frequency of stimuli to manipulate aspects of novelty. As we described, cross-sensory cue-target experiments might provide a generic platform to flexibly study several of these effects. 
If olfaction is in fact primarily an alarm system used to detect potential threats in the environment, as is often claimed, it is useful to reflect on what data would support this conclusion. Whether or not threat detection is the prioritized function of olfaction, neither typical laboratory experiments nor simulated breakfast scenarios are likely to provide strong evidence of such a notion. In the wild, or in our homes, sudden unexpected odors would alert us to the presence of something unusual and potentially dangerous. Both in research that supports the misfit account and in research that supports our predictive coding account, participants are matching odors to other, previously encountered odors, or to verbal or visual representations of these odors. It is challenging to experimentally assess human perceptual mechanisms used to detect olfactory threats in the wild. But if the ecological demands have shaped our olfactory abilities, we should see evidence of their presence even in well-controlled experimental conditions. The argument that all olfactory research needs to be conducted in situations that closely resemble ecological situations (e.g. Köster et al., 2014), is in our view mistaken. As we have argued elsewhere, ecological scenarios are informative, but often insufficient to understand the nature of the olfactory system, and carefully controlled experiments are thus indispensable (Olofsson and Pierzchajlo, 2021).

In future research, different types of olfactory novelty and related concepts should be carefully operationalized and compared. For instance, a stimulus could be familiar and expected: I buy a chocolate bar that I like, open it, and eat it. A stimulus could also be familiar but unexpected: Due to a mix-up in the chocolate factory, what I am eating has a different flavor than I expected, but it is a flavor I am familiar with. A stimulus could also be unfamiliar but expected: I knowingly eat a type of chocolate I never tried before. Finally, a stimulus could be unfamiliar and unexpected: I eat a chocolate flavor I have never tried before, mistaking it for a familiar flavor. Novelty and expectation violation can be dissociated, and a general conclusion from this overview is a recommendation to design research paradigms knowing exactly what operationalizations we are using. Furthermore, we should only accept theoretical claims of broad generalizability if they are supported by many converging pieces of experimental data. Broad theoretical claims, if they are to be considered true, should generate support from a variety of operationalizations of the key constructs, which is unfortunately rare in psychology (Yarkoni, 2020).

\section{Conclusion}


Olfaction is often claimed to prioritize novel stimuli. Attempts to provide evidence of such a notion are, in our view, fraught with shortcomings related to the small set of conditions under which novelty appears to be prioritized, problems regarding definitions of the novelty concept, and lack of consideration of experimental designs that would arguably be suitable to test the generalizability of novelty detection. Instead of novelty detection, we argue that olfactory processing is heavily influenced by predictions that can be effectively elicited by contextual cues, for example in audio-visual senses, and that facilitate rapid olfactory processing when the odors match the preceding cues. Odor recognition memory research does not provide support for enhanced processing of novel stimuli but instead suggests that previously encountered or known odors are better remembered. Research on behavioral and cortical processing of odors should focus on the role of predictions and prediction errors. Such an experimental framework, carefully dissociating how olfaction is affected by different forms of predictions and familiarity aspects, would facilitate a better understanding of human olfaction by emphasizing the profound roles that non-olfactory senses play in olfactory perception and cognition. 


\section{Reference}

Albright, T. D. (1989). Centrifugal directional bias in the middle temporal visual area (MT) of the macaque. Visual Neuroscience, 2(2), 177-188. https://doi.org/10.1017/s0952523800012037

Andersson, L., Sandberg, P., Olofsson, J. K., \& Nordin, S. (2018). Effects of Task Demands on Olfactory, Auditory, and Visual Event-Related Potentials Suggest Similar Top-Down Modulation Across Senses. Chemical Senses, 43(2), 129-134. https://doi.org/10.1093/chemse/bjx082

Cain, W. S. (1979). To know with the nose: Keys to odor identification. Science, 203(4379), 467-470. https://doi.org/10.1126/science.760202

Cain, W. S., de Wijk, R., Lulejian, C., Schiet, F., \& See, L.-C. (1998). Odor Identification: Perceptual and Semantic Dimensions. Chemical Senses, 23(3), 309-326. https://doi.org/10.1093/chemse/23.3.309

Clark, A. (2013). Whatever next? Predictive brains, situated agents, and the future of cognitive science. Behavioral and Brain Sciences, 36(3), 181-204. https://doi.org/10.1017/S0140525X12000477

Cloherty, S. L., Hughes, N. J., Hietanen, M. A., Bhagavatula, P. S., Goodhill, G. J., \& Ibbotson, M. R. (2016). Sensory experience modifies feature map relationships in visual cortex. ELife, 5, e13911. https://doi.org/10.7554/eLife.13911

Cornell Kärnekull, S., Jönsson, F. U., Willander, J., Sikström, S., \& Larsson, M. (2015). LongTerm Memory for Odors: Influences of Familiarity and Identification Across 64 Days. Chemical Senses, 40(4), 259-267. https://doi.org/10.1093/chemse/bjv003

Dalton, P. (2000). Psychophysical and Behavioral Characteristics of Olfactory Adaptation. Chemical Senses, 25(4), 487-492. https://doi.org/10.1093/chemse/25.4.487 
de Lange, F. P., Heilbron, M., \& Kok, P. (2018). How Do Expectations Shape Perception? Trends in Cognitive Sciences, 22(9), 764-779. https://doi.org/10.1016/j.tics.2018.06.002

Distler, C., Boussaoud, D., Desimone, R., \& Ungerleider, L. G. (1993). Cortical connections of inferior temporal area TEO in macaque monkeys. The Journal of Comparative Neurology, 334(1), 125-150. https://doi.org/10.1002/cne.903340111

Egner, T., Monti, J. M., \& Summerfield, C. (2010). Expectation and Surprise Determine Neural Population Responses in the Ventral Visual Stream. Journal of Neuroscience, 30(49), 16601-16608. https://doi.org/10.1523/JNEUROSCI.2770-10.2010

Elsberg, C. (1935). Olfactory fatigue. Bull Neurol Inst, 4, 479-495.

Engen, T., \& Ross, B. M. (1973). Long-term memory of odors with and without verbal descriptions. Journal of Experimental Psychology, 100(2), 221-227. https://doi.org/10.1037/h0035492

Fahy, F. L., Riches, I. P., \& Brown, M. W. (1993). Neuronal activity related to visual recognition memory: Long-term memory and the encoding of recency and familiarity information in the primate anterior and medial inferior temporal and rhinal cortex. Experimental Brain Research, 96(3), 457-472. https://doi.org/10.1007/BF00234113

Gottfried, J. A., Winston, J. S., \& Dolan, R. J. (2006). Dissociable Codes of Odor Quality and Odorant Structure in Human Piriform Cortex. Neuron, 49(3), 467-479. https://doi.org/10.1016/j.neuron.2006.01.007

Gregory, R. L. (1980). Perceptions as Hypotheses. Philosophical Transactions of the Royal Society of London. Series B, Biological Sciences, 290(1038), 181-197. JSTOR.

Helmholtz, Hermann von-Treatise on physiological optics, Bd. 3: The perceptions of vision. (1860). https://echo.mpiwg- 
berlin.mpg.de/ECHOdocuView?url=/permanent/library/HS7FH69N/pageimg\&pn=5\& mode=imagepath

Herrick, C. J. (1933). The Functions of the Olfactory Parts of the Cerebral Cortex. Proceedings of the National Academy of Sciences, 19(1), 7-14. https://doi.org/10.1073/pnas.19.1.7

Hörberg, T., Larsson, M., Ekström, I., Sandöy, C., Lundén, P., \& Olofsson, J. K. (2020). Olfactory Influences on Visual Categorization: Behavioral and ERP Evidence. Cerebral Cortex, 30(7), 4220-4237. https://doi.org/10.1093/cercor/bhaa050

Howard, J. D., Plailly, J., Grueschow, M., Haynes, J.-D., \& Gottfried, J. A. (2009). Odor quality coding and categorization in human posterior piriform cortex. Nature Neuroscience, 12(7), 932-938. https://doi.org/10.1038/nn.2324

Jonides, J., \& Yantis, S. (1988). Uniqueness of abrupt visual onset in capturing attention. Perception \& Psychophysics, 43(4), 346-354. https://doi.org/10.3758/BF03208805

Jönsson, F. U., Olsson, H., \& Olsson, M. J. (2005). Odor emotionality affects the confidence in odor naming. Chemical Senses, 30(1), 29-35.

https://doi.org/10.1093/chemse/bjh254

Jönsson, F. U., \& Olsson, M. J. (2003). Olfactory metacognition. Chemical Senses, 28(7), 651658. https://doi.org/10.1093/chemse/bjg058

Kafkas, A., Migo, E. M., Morris, R. G., Kopelman, M. D., Montaldi, D., \& Mayes, A. R. (2017). Material Specificity Drives Medial Temporal Lobe Familiarity But Not Hippocampal Recollection: FAMILIARITY-BASED RECOGNITION IN THE MTL. Hippocampus, 27(2), 194-209. https://doi.org/10.1002/hipo.22683

Kafkas, A., \& Montaldi, D. (2018). How do memory systems detect and respond to novelty? Neuroscience Letters, 680, 60-68. https://doi.org/10.1016/j.neulet.2018.01.053 
Khan, R. M., Luk, C.-H., Flinker, A., Aggarwal, A., Lapid, H., Haddad, R., \& Sobel, N. (2007). Predicting Odor Pleasantness from Odorant Structure: Pleasantness as a Reflection of the Physical World. Journal of Neuroscience, 27(37), 10015-10023. https://doi.org/10.1523/JNEUROSCI.1158-07.2007

Knight, R. T. (1996). Contribution of human hippocampal region to novelty detection. Nature, 383(6597), 256-259. https://doi.org/10.1038/383256a0

Kok, P., Jehee, J. F. M., \& de Lange, F. P. (2012). Less Is More: Expectation Sharpens Representations in the Primary Visual Cortex. Neuron, 75(2), 265-270. https://doi.org/10.1016/j.neuron.2012.04.034

Köster, E. P., \& de Wijk, R. A. (1991). Olfactory Adaptation. In D. G. Laing, R. L. Doty, \& W. Breipohl (Eds.), The Human Sense of Smell (pp. 199-215). Springer. https://doi.org/10.1007/978-3-642-76223-9_10

Köster, E. P., Møller, P., \& Mojet, J. (2014). A “Misfit" Theory of Spontaneous Conscious Odor Perception (MITSCOP): Reflections on the role and function of odor memory in everyday life. Frontiers in Psychology, 5. https://doi.org/10.3389/fpsyg.2014.00064

Koster, M. A. (2004). Incidental Learning and Memory for Three Basic Tastes in Food. Chemical Senses, 29(5), 441-453. https://doi.org/10.1093/chemse/bjh047

Kumaran, D., \& Maguire, E. A. (2007). Match mismatch processes underlie human hippocampal responses to associative novelty. The Journal of Neuroscience: The Official Journal of the Society for Neuroscience, 27(32), 8517-8524. https://doi.org/10.1523/JNEUROSCI.1677-07.2007

Kuraoka, K., \& Nakamura, K. (2007). Responses of Single Neurons in Monkey Amygdala to Facial and Vocal Emotions. Journal of Neurophysiology, 97(2), 1379-1387. https://doi.org/10.1152/jn.00464.2006 
Laing, \& Francis, G. W. (1989). The capacity of humans to identify odors in mixtures.

Physiology \& Behavior, 46(5), 809-814. https://doi.org/10.1016/00319384(89)90041-3

Laing, \& MacLeod, P. (1992). Reaction time for the recognition of odor quality. Chemical Senses, 17(3), 337-346. https://doi.org/10.1093/chemse/17.3.337

Laureati, M., Morin-Audebrand, L., Pagliarini, E., Sulmont-Rossé, C., Köster, E. P., \& Mojet, J. (2008). Food memory and its relation with age and liking: An incidental learning experiment with children, young and elderly people. Appetite, 51(2), 273-282. https://doi.org/10.1016/j.appet.2008.02.019

Lavenex, P., \& Amaral, D. G. (2000). Hippocampal-neocortical interaction: A hierarchy of associativity. Hippocampus, 10(4), 420-430. https://doi.org/10.1002/10981063(2000)10:4<420::AID-HIPO8>3.0.CO;2-5

Li, L., Miller, E. K., \& Desimone, R. (1993). The representation of stimulus familiarity in anterior inferior temporal cortex. Journal of Neurophysiology, 69(6), 1918-1929. Livermore, A., \& Laing. (1996). Influence of Training and Experience on the Perception of Multicomponent Odor Mixtures. 22(2), 11.

MacKay, D. M. (1956). The Epistemological Problem for Automata. https://doi.org/10.1515/9781400882618-012

Markowitsch, H. J., Craik, F. E., Habib, R., \& Houle, S. (1996). Novelty and familiarity activations in PET studies of memory encoding and retrieval. Cerebral Cortex (New York, N.Y.: 1991), 6(1), 71-79. https://doi.org/10.1093/cercor/6.1.71

Mojet, J., \& Köster, E. P. (2005). Sensory memory and food texture. Food Quality and Preference, 16(3), 251-266. https://doi.org/10.1016/j.foodqual.2004.04.017 
Moller, P., Mojet, J., \& Koster, E. P. (2007). Incidental and Intentional Flavor Memory in Young and Older Subjects. Chemical Senses, 32(6), 557-567. https://doi.org/10.1093/chemse/bjm026

Morin-Audebrand, L., Mojet, J., Chabanet, C., Issanchou, S., Møller, P., Köster, E., \& SulmontRossé, C. (2012). The role of novelty detection in food memory. Acta Psychologica, 139(1), 233-238. https://doi.org/10.1016/j.actpsy.2011.10.003

Morin-Audebrand, L., Sulmont-Rossé, C., \& Issanchou, S. (2006). Impact of hedonic appreciation on memory for custard dessert. Chemical Senses, 31. https://academic.oup.com/chemse/article-lookup/doi/10.1093/chemse/bjl029 Moulton, D. G. (1967). Olfaction in Mammals. American Zoologist, 7(3), 421-429. https://doi.org/10.1093/icb/7.3.421

Neisser, U. (1967). Cognitive psychology. Appleton-Century-Crofts.

Nordin, S., Monsch, A. U., \& Murphy, C. (1995). Unawareness of Smell Loss in Normal Aging and Alzheimer's Disease: Discrepancy between Self-Reported and Diagnosed Smell Sensitivity. The Journals of Gerontology Series B: Psychological Sciences and Social Sciences, 50B(4), P187-P192. https://doi.org/10.1093/geronb/50B.4.P187

Öhman, A., Lundqvist, D., \& Esteves, F. (2001). The face in the crowd revisited: A threat advantage with schematic stimuli. Journal of Personality and Social Psychology, 80(3), 381-396. https://doi.org/10.1037/0022-3514.80.3.381

Oleszkiewicz, A., Schriever, V. A., Croy, I., Hähner, A., \& Hummel, T. (2019). Updated Sniffin' Sticks normative data based on an extended sample of 9139 subjects. European Archives of Oto-Rhino-Laryngology: Official Journal of the European Federation of Oto-Rhino-Laryngological Societies (EUFOS): Affiliated with the German Society for 
Oto-Rhino-Laryngology - Head and Neck Surgery, 276(3), 719-728.

https://doi.org/10.1007/s00405-018-5248-1

Oliva, A., \& Torralba, A. (2007). The role of context in object recognition. Trends in Cognitive Sciences, 11(12), 520-527. https://doi.org/10.1016/j.tics.2007.09.009

Olofsson, J. K. (2014). Time to smell: A cascade model of human olfactory perception based on response-time (RT) measurement. Frontiers in Psychology, 5. https://doi.org/10.3389/fpsyg.2014.00033

Olofsson, J. K., Bowman, N. E., \& Gottfried, J. A. (2013). High and low roads to odor valence? A choice response-time study. Journal of Experimental Psychology: Human Perception and Performance, 39(5), 1205-1211. https://doi.org/10.1037/a0033682

Olofsson, J. K., Bowman, N. E., Khatibi, K., \& Gottfried, J. A. (2012). A Time-Based Account of the Perception of Odor Objects and Valences: Psychological Science. https://doi.org/10.1177/0956797612441951

Olofsson, J. K., \& Gottfried, J. A. (2015). The muted sense: Neurocognitive limitations of olfactory language. Trends in Cognitive Sciences, 19(6), 314-321. https://doi.org/10.1016/j.tics.2015.04.007

Olofsson, J. K., Hurley, R. S., Bowman, N. E., Bao, X., Mesulam, M.-M., \& Gottfried, J. A. (2014). A Designated Odor-Language Integration System in the Human Brain. Journal of Neuroscience, 34(45), 14864-14873. https://doi.org/10.1523/JNEUROSCI.224714.2014

Olofsson, J. K., \& Pierzchajlo, S. (2021). Olfactory Language: Context Is Everything. Trends in Cognitive Sciences. https://doi.org/10.1016/j.tics.2021.02.004 
Olofsson, J. K., \& Polich, J. (2007). Affective visual event-related potentials: Arousal, repetition, and time-on-task. Biological Psychology, 75(1), 101-108. https://doi.org/10.1016/j.biopsycho.2006.12.006

Olofsson, J. K., Syrjänen, E., Ekström, I., Larsson, M., \& Wiens, S. (2018). “Fast" versus "slow" word integration of visual and olfactory objects: EEG biomarkers of decision speed variability. Behavioral Neuroscience, 132(6), 587-594.

https://doi.org/10.1037/bne0000266

Paller, K. (1994). The Neural Substrates of Cognitive Event-Related Potentials: A Review of Animal Models of P3. In Cognitive Electrophysiology (pp. 300-333). Birkhauser, Boston, Massachusetts.

Pierzchajlo, S., Jernsäther, T., Fontana, L., Zampini, M., \& Olofsson, J. (2021). Olfactory identification is more Reliant than Visual Identification on Predictive Object Cues. Unpublished Manuscript.

Pierzchajlo, S., Jernsäther-Ohlsson, T., \& Olofsson, J. (2019). Do verbal object cues similarly shape integration of olfactory and visual targets? XXIXth Annual Meeting of the European Chemoreception Research Organization, Trieste, Italy.

Porada, D. K., Regenbogen, C., Seubert, J., Freiherr, J., \& Lundström, J. N. (2019). Multisensory Enhancement of Odor Object Processing in Primary Olfactory Cortex. Neuroscience, 418, 254-265. https://doi.org/10.1016/j.neuroscience.2019.08.040 Ranganath, C., \& Rainer, G. (2003). Neural mechanisms for detecting and remembering novel events. Nature Reviews Neuroscience, 4(3), 193-202. https://doi.org/10.1038/nrn1052 
Rao, R. P. N., \& Ballard, D. H. (1999). Predictive coding in the visual cortex: A functional interpretation of some extra-classical receptive-field effects. Nature Neuroscience, 2(1), 79-87. https://doi.org/10.1038/4580

Ribeiro, P. F. M., Manger, P. R., Catania, K. C., Kaas, J. H., \& Herculano-Houzel, S. (2014). Greater addition of neurons to the olfactory bulb than to the cerebral cortex of eulipotyphlans but not rodents, afrotherians or primates. Frontiers in Neuroanatomy, 8. https://doi.org/10.3389/fnana.2014.00023

Ringo, J. L. (1996). Stimulus specific adaptation in inferior temporal and medial temporal cortex of the monkey. Behavioural Brain Research, 76(1), 191-197. https://doi.org/10.1016/0166-4328(95)00197-2

Robinson, D. A. (1968). Eye Movement Control in Primates. Science, 161(3847), 1219-1224. https://doi.org/10.1126/science.161.3847.1219

Sanchez-Vives, M. V., Nowak, L. G., \& McCormick, D. A. (2000). Cellular Mechanisms of LongLasting Adaptation in Visual Cortical Neurons In Vitro. Journal of Neuroscience, 20(11), 4286-4299. https://doi.org/10.1523/JNEUROSCI.20-11-04286.2000

Sander, K., \& Scheich, H. (2001). Auditory perception of laughing and crying activates human amygdala regardless of attentional state. Cognitive Brain Research, 12(2), 181-198. https://doi.org/10.1016/S0926-6410(01)00045-3

Saygin, Z. M., Osher, D. E., Koldewyn, K., Reynolds, G., Gabrieli, J. D. E., \& Saxe, R. R. (2012). Anatomical connectivity patterns predict face selectivity in the fusiform gyrus. Nature Neuroscience, 15(2), 321-327. https://doi.org/10.1038/nn.3001

Schab, F. R. (1991). Odor memory: Taking stock. Psychological Bulletin, 109(2), 242-251. https://doi.org/10.1037/0033-2909.109.2.242 
Singer, W., \& Gray, C. M. (1995). Visual Feature Integration and the Temporal Correlation Hypothesis. Annual Review of Neuroscience, 18(1), 555-586. https://doi.org/10.1146/annurev.ne.18.030195.003011

Sohal, V. S., \& Hasselmo, M. E. (2000). A model for experience-dependent changes in the responses of inferotemporal neurons. Network: Computation in Neural Systems, 11(3), 169-190. https://doi.org/10.1088/0954-898X_11_3_301

Staresina, B. P., Fell, J., Do Lam, A. T. A., Axmacher, N., \& Henson, R. N. (2012). Memory signals are temporally dissociated in and across human hippocampus and perirhinal cortex. Nature Neuroscience, 15(8), 1167-1173. https://doi.org/10.1038/nn.3154

Sulmont-Rossé, C., Møller, P., Issanchou, S., \& Köster, E. P. (2008). Effect of Age and Food Novelty on Food Memory. Chemosensory Perception, 1(3), 199-209. https://doi.org/10.1007/s12078-008-9025-1

Sutton, S., Braren, M., Zubin, J., \& John, E. R. (1965). Evoked-Potential Correlates of Stimulus Uncertainty. Science, 150(3700), 1187-1188. https://doi.org/10.1126/science.150.3700.1187

Tanaka, K. (1996). Inferotemporal Cortex and Object Vision. Annual Review of Neuroscience, 19(1), 109-139. https://doi.org/10.1146/annurev.ne.19.030196.000545

Thomas-Danguin, T., Sinding, C., Romagny, S., El Mountassir, F., Atanasova, B., Le Berre, E., Le Bon, A.-M., \& Coureaud, G. (2014). The perception of odor objects in everyday life: A review on the processing of odor mixtures. Frontiers in Psychology, 5. https://doi.org/10.3389/fpsyg.2014.00504

Treisman, A., \& Gormican, S. (1988). Feature Analysis in Early Vision: Evidence From Search Asymmetries. 34. 
Tulving, E. (2010). How do brains detect novelty? In Memory, aging and the brain: $A$ Festschrift in honour of Lars-Göran Nilsson (pp. 92-112). Psychology Press.

Tulving, E., \& Kroll, N. (1995). Novelty assessment in the brain and long-term memory encoding. Psychonomic Bulletin \& Review, 2(3), 387-390. https://doi.org/10.3758/BF03210977

Tulving, E., Markowitsch, H. J., Kapur, S., Habib, R., \& Houle, S. (1994). Novelty encoding networks in the human brain: Positron emission tomography data. Neuroreport: An International Journal for the Rapid Communication of Research in Neuroscience, 5(18), 2525-2528. https://doi.org/10.1097/00001756-199412000-00030

Tulving, E., \& Rosenbaum, R. S. (2006). What do explanations of the distinctiveness effect need to explain? In Distinctiveness and memory (pp. 407-423). Oxford University Press. https://doi.org/10.1093/acprof:oso/9780195169669.003.0018

Van Essen, D., Anderson, C., \& Felleman, D. (1992). Information processing in the primate visual system: An integrated systems perspective. Science, 255(5043), 419-423. https://doi.org/10.1126/science.1734518

Wilson, D. A., \& Stevenson, R. J. (2003). Olfactory perceptual learning: The critical role of memory in odor discrimination. Neuroscience \& Biobehavioral Reviews, 27(4), 307328. https://doi.org/10.1016/S0149-7634(03)00050-2

Wilson, D. A., \& Sullivan, R. M. (2011). Cortical Processing of Odor Objects. Neuron, 72(4), 506-519. https://doi.org/10.1016/j.neuron.2011.10.027

Yarkoni, T. (2020). The generalizability crisis. The Behavioral and Brain Sciences, 1-37. https://doi.org/10.1017/S0140525X20001685

Yeshurun, Y., \& Sobel, N. (2010). An Odor is Not Worth a Thousand Words: From Multidimensional Odors to Unidimensional Odor Objects. Annual Review of 
Psychology, 61(1), 219-241.

https://doi.org/10.1146/annurev.psych.60.110707.163639

Yuille, A., \& Kersten, D. (2006). Vision as Bayesian inference: Analysis by synthesis? Trends in Cognitive Sciences, 10(7), 301-308. https://doi.org/10.1016/j.tics.2006.05.002

Zald, D. H., \& Pardo, J. V. (2002). The Neural Correlates of Aversive Auditory Stimulation. Neurolmage, 16(3), 746-753. https://doi.org/10.1006/nimg.2002.1115

Zarzo, M. (2008). PSYCHOLOGIC DIMENSIONS IN THE PERCEPTION OF EVERYDAY ODORS: PLEASANTNESS AND EDIBILITY. Journal of Sensory Studies, 23(3), 354-376. https://doi.org/10.1111/j.1745-459X.2008.00160.x

Zelano, C., Mohanty, A., \& Gottfried, J. A. (2011). Olfactory Predictive Codes and Stimulus Templates in Piriform Cortex. Neuron, 72(1), 178-187. https://doi.org/10.1016/j.neuron.2011.08.010

Zhou, G., Lane, G., Noto, T., Arabkheradmand, G., Gottfried, J. A., Schuele, S. U., Rosenow, J. M., Olofsson, J. K., Wilson, D. A., \& Zelano, C. (2019). Human olfactory-auditory integration requires phase synchrony between sensory cortices. Nature Communications, 10(1), 1168. https://doi.org/10.1038/s41467-019-09091-3 